\title{
TRUNCATED VARIATION, UPWARD TRUNCATED VARIATION AND DOWNWARD TRUNCATED VARIATION OF BROWNIAN MOTION WITH DRIFT-THEIR MEAN VALUE AND THEIR APPLICATIONS
}

\author{
RAFAє M. ŁOCHOWSKI \\ Department of Mathematics and Mathematical Economics, Warsaw School of Economics \\ Al. Niepodległości 162, 02-554 Warszawa, Poland \\ E-mail: rlocho@sgh.waw.pl
}

Abstract. In [6] for $c>0$ we defined the truncated variation, $T V_{\mu}^{c}$, of a Brownian motion with drift, $W_{t}=B_{t}+\mu t, t \geq 0$, where $\left(B_{t}\right)$ is a standard Brownian motion. In this article we define two related quantities: the upward truncated variation

$$
U T V_{\mu}^{c}[a, b]=\sup _{n} \sup _{a \leq t_{1}<s_{1}<\ldots<t_{n}<s_{n} \leq b} \sum_{i=1}^{n} \max \left\{W_{s_{i}}-W_{t_{i}}-c, 0\right\}
$$

and, analogously, the downward truncated variation

$$
D T V_{\mu}^{c}[a, b]=\sup _{n} \sup _{a \leq t_{1}<s_{1}<\ldots<t_{n}<s_{n} \leq b} \sum_{i=1}^{n} \max \left\{W_{t_{i}}-W_{s_{i}}-c, 0\right\} .
$$

We prove that the exponential moments of the above quantities are finite (in contrast to the regular variation, corresponding to $c=0$, which is infinite almost surely). We present estimates of the expected value of $U T V_{\mu}^{c}$ up to universal constants.

As an application we give some estimates of the maximal possible gain from trading a financial asset in the presence of flat commission (proportional to the value of the transaction) when the dynamics of the prices of the asset follows a geometric Brownian motion process. In the presented estimates the upward truncated variation appears naturally.

1. Introduction. Let $\left(B_{t}, t \geq 0\right)$ be a standard Brownian motion, and $W_{t}=B_{t}+\mu t$ be a Brownian motion with drift $\mu$.

2010 Mathematics Subject Classification: Primary 60G15.

Key words and phrases: Brownian motion, variation.

The paper is in final form and no version of it will be published elsewhere. 
In [6] the truncated variation at the level $c>0$ of the Brownian motion with drift $\mu$ on the interval $[a, b]$ was defined as

$$
T V_{\mu}^{c}[a, b]:=\sup _{n} \sup _{a \leq t_{1} \leq \cdots \leq t_{n} \leq b} \sum_{i=1}^{n-1} \max \left\{\left|W_{t_{i+1}}-W_{t_{i}}\right|-c, 0\right\} .
$$

(Technical remark: for $a>b$ we set $T V_{\mu}^{c}[a, b]=0$.)

There were also proved estimates of $\mathbf{E} T V_{\mu}^{c}[0, T]$ up to universal constants. Using similar techniques as in [6] we will prove existence of finite exponential moments of $T V_{\mu}^{c}[0, T], \mathbf{E} \exp \left(\alpha T V_{\mu}^{c}[0, T]\right)$, for any $\alpha, T>0$.

Further we will consider two related quantities:

- the upward truncated variation, defined as

$$
U T V_{\mu}^{c}[a, b]:=\sup _{n} \sup _{a \leq t_{1}<s_{1}<\cdots<t_{n}<s_{n} \leq b} \sum_{i=1}^{n} \max \left\{W_{s_{i}}-W_{t_{i}}-c, 0\right\},
$$

- analogously, the downward truncated variation, defined as

$$
D T V_{\mu}^{c}[a, b]:=\sup _{n} \sup _{a \leq t_{1}<s_{1}<\cdots<t_{n}<s_{n} \leq b} \sum_{i=1}^{n} \max \left\{W_{t_{i}}-W_{s_{i}}-c, 0\right\} .
$$

It is easy to see that all three above defined quantities have the following properties, which we state only for the truncated variation:

- shift invariance property in distributions:

$$
\mathcal{L}\left(T V_{\mu}^{c}[a, b]\right)=\mathcal{L}\left(T V_{\mu}^{c}[a+\Delta, b+\Delta]\right),
$$

- superadditivity: for any numbers $a \leq a_{1}<a_{2}<\cdots<a_{n} \leq b$

$$
T V_{\mu}^{c}[a, b] \geq \sum_{i=1}^{n-1} T V_{\mu}^{c}\left[a_{i}, a_{i+1}\right] .
$$

It is also easy to see that the following relations hold

$$
\begin{aligned}
T V_{\mu}^{c}[0, T] & \geq U T V_{\mu}^{c}[0, T], \\
T V_{\mu}^{c}[0, T] & \geq D T V_{\mu}^{c}[0, T], \\
T V_{\mu}^{c}[0, T] & \leq U T V_{\mu}^{c}[0, T]+D T V_{\mu}^{c}[0, T], \\
\mathcal{L}\left(U T V_{\mu}^{c}[0, T]\right) & =\mathcal{L}\left(D T V_{-\mu}^{c}[0, T]\right) .
\end{aligned}
$$

By (3) all estimates proved for the upward truncated variation have analogs for the downward truncated variation.

Analogously as in [6] we will prove some estimates of $\mathbf{E} U T V_{\mu}^{c}[0, T]$ (and thus for $\left.\mathbf{E} D T V_{\mu}^{c}[0, T]\right)$ up to universal constants. Unfortunately, the estimates involve the expected values of some other related variables.

REMARK. In order to shorten the proofs we did not put much stress on obtaining the best possible constants.

REMARK. K. Oleszkiewicz pointed out that it would be also interesting to have estimates for higher moments of the defined quantities. However, the author presumes that other methods than those used in this paper are needed to obtain such estimates. 
Remark. A. N. Chuprunov pointed out to the author that it would be also interesting to have estimates of the quadratic truncated variation, which one may define as

$$
Q T V_{\mu}^{c}[a, b]:=\sup _{n} \sup _{a \leq t_{1} \leq \cdots \leq t_{n} \leq b} \sum_{i=1}^{n-1} \max \left\{\left|W_{t_{i+1}}-W_{t_{i}}\right|^{2}-c^{2}, 0\right\} .
$$

REMARK. A similar concept of truncation (or shrinking) of random variables on Hilbert spaces was investigated by Z. J. Jurek in a series of papers beginning with [2], 3], which now evolved into the theory of s-selfdecomposable distributions (see e.g. 4]).

2. Existence of exponential moments of truncated variation. Let us start with the existence of finite exponential moments of $T V_{\mu}^{c}[0, T]$. To prove this let us define

$$
T_{c}=\inf \left\{t \geq 0: \sup _{0 \leq s \leq t} W_{s} \geq W_{t}+c\right\},
$$

further let $T_{c}^{\text {sup }}$ be the last instant when the maximum of $W_{t}$ on $\left[0, T_{c}\right]$ is attained, and let $T_{c}^{\text {inf }} \leq T_{c}^{\text {sup }}$ be such that $W_{T_{c}^{\text {inf }}}=\inf _{0 \leq s \leq T_{c}^{\text {sup }}} W_{s}$.

Let us fix $\alpha>0$ and let $\delta>0$ be a small number such that

$$
1-\mathbf{E} \exp \left(\alpha \sup _{0 \leq t \leq T} W_{t}+\alpha c\right) P\left(T_{c}<\delta\right)>0 .
$$

By definition of $T_{c}$ and $T_{c}^{\text {inf }}$ we have $W_{T_{c}^{\text {inf }}}>-c$ and $W_{T_{c}^{\text {sup }}}-W_{T_{c}^{\text {inf }}}-c \leq W_{T_{c}^{\text {sup }}}$. Now, by Lemma 1, Lemma 2 in [6] and independence of $W_{t}-W_{T_{c}}, t \geq T_{c}$, and $T_{c}$ (strong Markov property of Brownian motion) for any $M>0$ we have

$$
\begin{aligned}
& \mathbf{E} \exp \left(\alpha T V_{\mu}^{c}[0, T] \wedge M\right) \leq \mathbf{E} \exp \left(\alpha W_{T_{c}^{\text {sup }}}+\alpha c+\alpha T V_{\mu}^{c}\left[T_{c}, T\right] \wedge M\right) \\
& \leq \mathbf{E} \exp \left(\alpha W_{T_{c}^{\text {sup }}}+\alpha c\right) \mathbf{E} \exp \left[\alpha T V_{\mu}^{c}\left[T_{c}, T\right] \wedge M ; T_{c}<\delta\right] \\
& \quad+\mathbf{E} \exp \left(\alpha W_{T_{c}^{\text {sup }}}+\alpha c\right) \mathbf{E} \exp \left[\alpha T V_{\mu}^{c}\left[T_{c}, T\right] \wedge M ; T_{c} \geq \delta\right] \\
& \leq \mathbf{E} \exp \left(\alpha W_{T_{c}^{\text {sup }}}+\alpha c\right) \mathbf{E} \exp \left[\alpha T V_{\mu}^{c}\left[T_{c}, T+T_{c}\right] \wedge M ; T_{c}<\delta\right] \\
& \quad+\mathbf{E} \exp \left(\alpha W_{T_{c}^{\text {sup }}}+\alpha c\right) \mathbf{E} \exp \left[\alpha T V_{\mu}^{c}\left[T_{c}, T+T_{c}-\delta\right] \wedge M ; T_{c} \geq \delta\right] \\
& \leq \mathbf{E} \exp \left(\alpha \sup _{0 \leq t \leq T} W_{t}+\alpha c\right) \mathbf{E} \exp \left(\alpha T V_{\mu}^{c}[0, T] \wedge M\right) P\left(T_{c}<\delta\right) \\
&+\mathbf{E} \exp \left(\alpha \sup _{0 \leq t \leq T} W_{t}+\alpha c\right) \mathbf{E} \exp \left(\alpha T V_{\mu}^{c}[0, T-\delta] \wedge M\right) P\left(T_{c} \geq \delta\right) .
\end{aligned}
$$

From the above we have

$$
\begin{aligned}
& \mathbf{E} \exp \left(\alpha T V_{\mu}^{c}[0, T] \wedge M\right) \\
& \quad \leq \frac{\mathbf{E} \exp \left(\alpha \sup _{0 \leq t \leq T} W_{t}+\alpha c\right) P\left(T_{c} \geq \delta\right)}{1-\mathbf{E} \exp \left(\alpha \sup _{0 \leq t \leq T} W_{t}+\alpha c\right) P\left(T_{c}<\delta\right)} \mathbf{E} \exp \left(\alpha T V_{\mu}^{c}[0, T-\delta] \wedge M\right) .
\end{aligned}
$$

Similarly

$$
\begin{aligned}
& \mathbf{E} \exp \left(\alpha T V_{\mu}^{c}[0, T-\delta] \wedge M\right) \\
& \quad \leq \frac{\mathbf{E} \exp \left(\alpha \sup _{0 \leq t \leq T} W_{t}+\alpha c\right) P\left(T_{c} \geq \delta\right)}{1-\mathbf{E} \exp \left(\alpha \sup _{0 \leq t \leq T} W_{t}+\alpha c\right) P\left(T_{c}<\delta\right)} \mathbf{E} \exp \left(\alpha T V_{\mu}^{c}[0, T-2 \delta] \wedge M\right) .
\end{aligned}
$$


Iterating and putting together the above inequalities we finally obtain

$$
\mathbf{E} \exp \left(\alpha T V_{\mu}^{c}[0, T] \wedge M\right) \leq\left(\frac{\mathbf{E} \exp \left(\alpha \sup _{0 \leq t \leq T} W_{t}+\alpha c\right) P\left(T_{c} \geq \delta\right)}{1-\mathbf{E} \exp \left(\alpha \sup _{0 \leq t \leq T} W_{t}+\alpha c\right) P\left(T_{c}<\delta\right)}\right)^{\lceil T / \delta\rceil}
$$

Letting $M \rightarrow \infty$ we get $\mathbf{E} \exp \left(\alpha T V_{\mu}^{c}[0, T]\right)<+\infty$.

By (1) and (2) we obtain the finiteness of exponential moments of $U T V_{\mu}^{c}[0, T]$ and $D T V_{\mu}^{c}[0, T]$ as well.

\section{Estimates of expected value of upward and downward truncated variation}

3.1. Preparatory lemmas. In order to obtain estimates of $\mathbf{E} U T V_{\mu}^{c}[0, T]$ (and analogously $\mathbf{E} D T V_{\mu}^{c}[0, T]$ ) we will use similar techniques as in [6]. Due to typographical reasons let us introduce the notation $\max \{x, 0\}=:(x)_{+}$.

We will need the following analog of Lemma 2 from [6]:

LEMMA 3.1. We have the following identity

$$
U T V_{\mu}^{c}[0, T]=\sup _{0 \leq t<s \leq T_{c} \wedge T}\left(W_{s}-W_{t}-c\right)_{+}+U T V_{\mu}^{c}\left[T_{c}, T\right] .
$$

Proof. Let $0 \leq t_{1}<s_{1}<t_{2}<s_{2}<\cdots<t_{n}<s_{n} \leq T$. We will prove that

$$
\sum_{i=1}^{n}\left(W_{s_{i}}-W_{t_{i}}-c\right)_{+} \leq \sup _{0 \leq t<s \leq T_{c} \wedge T}\left(W_{s}-W_{t}-c\right)_{+}+U T V_{\mu}^{c}\left[T_{c}, T\right] .
$$

Let $n_{0}$ be the greatest number such that $s_{n_{0}}<T_{c}$ and let us assume that $n_{0}<n$ and $t_{n_{0}+1}<T_{c}$.

Let us consider several cases.

- $W_{t_{n_{0}+1}} \geq W_{T_{c}}$. In this case

$$
\left(W_{s_{n_{0}+1}}-W_{t_{n_{0}+1}}-c\right)_{+} \leq\left(W_{s_{n_{0}+1}}-W_{T_{c}}-c\right)_{+}
$$

and

$$
\begin{aligned}
\sum_{i=1}^{n}\left(W_{s_{i}}-W_{t_{i}}-c\right)_{+} & \leq \sum_{i=1}^{n_{0}}\left(W_{s_{i}}-W_{t_{i}}-c\right)_{+}+\left(W_{s_{n_{0}+1}}-W_{T_{c}}-c\right)_{+} \\
& +\sum_{i=n_{0}+2}^{n}\left(W_{s_{i}}-W_{t_{i}}-c\right)_{+} \cdot
\end{aligned}
$$

- $W_{t_{n_{0}+1}}<W_{T_{c}}$ and $W_{s_{n_{0}+1}} \leq W_{T_{c}^{\text {sup }}}$. In this case $t_{n_{0}+1}<T_{c}^{\text {sup }}$ (since for $T_{c}^{\text {sup }}<$ $\left.t<T_{c}, W_{t}>W_{T_{c}}\right)$ so

$$
\left(W_{s_{n_{0}+1}}-W_{t_{n_{0}+1}}-c\right)_{+} \leq\left(W_{T_{c}^{\text {sup }}}-W_{t_{n_{0}+1}}-c\right)_{+}
$$

and

$$
\begin{aligned}
\sum_{i=1}^{n}\left(W_{s_{i}}-W_{t_{i}}-c\right)_{+} & \leq \sum_{i=1}^{n_{0}}\left(W_{s_{i}}-W_{t_{i}}-c\right)_{+}+\left(W_{T_{c}^{\text {sup }}}-W_{t_{n_{0}+1}}-c\right)_{+} \\
& +\sum_{i=n_{0}+2}^{n}\left(W_{s_{i}}-W_{t_{i}}-c\right)_{+} \cdot
\end{aligned}
$$


- $W_{t_{n_{0}+1}}<W_{T_{c}}$ and $W_{s_{n_{0}+1}}>W_{T_{c}^{\text {sup }}}=W_{T_{c}}+c$. In this case

$$
\begin{aligned}
\left(W_{s_{n_{0}+1}}-W_{t_{n_{0}+1}}-c\right)_{+} & =W_{s_{n_{0}+1}}-W_{t_{n_{0}+1}}-c \\
& =W_{T_{c}^{\text {sup }}}-W_{t_{n_{0}+1}}-c+W_{s_{n_{0}+1}}-W_{T_{c}^{\text {sup }}} \\
& =W_{T_{c}^{\text {sup }}}-W_{t_{n_{0}+1}}-c+W_{s_{n_{0}+1}}-W_{T_{c}}-c \\
& =\left(W_{T_{c}^{\text {sup }}}-W_{t_{n_{0}+1}}-c\right)_{+}+\left(W_{s_{n_{0}+1}}-W_{T_{c}}-c\right)_{+}
\end{aligned}
$$

and

$$
\begin{aligned}
\sum_{i=1}^{n}\left(W_{s_{i}}-W_{t_{i}}-c\right)_{+} & \leq \sum_{i=1}^{n_{0}}\left(W_{s_{i}}-W_{t_{i}}-c\right)_{+}+\left(W_{T_{c}^{\text {sup }}}-W_{t_{n_{0}+1}}-c\right)_{+} \\
& +\left(W_{s_{n_{0}+1}}-W_{T_{c}}-c\right)_{+}+\sum_{i=n_{0}+2}^{n}\left(W_{s_{i}}-W_{t_{i}}-c\right)_{+} .
\end{aligned}
$$

Thus for $t_{n_{0}+1}<T_{c}$ inequality (6), (7) or (8) holds and we may assume, adding in the case $t_{n_{0}+1}<T_{c}$ new terms in the partition and renaming the old ones, that

$$
\begin{aligned}
0 & \leq t_{1}<s_{1}<\cdots<t_{n_{0}}<s_{n_{0}} \leq T_{c}, \\
T_{c} & \leq t_{n_{0}+1}<s_{n_{0}+1}<\cdots<t_{n}<s_{n} \leq T .
\end{aligned}
$$

In order to prove (5) without loss of generality we may assume that for any $1 \leq i \leq n_{0}$, $\left(W_{s_{i}}-W_{t_{i}}-c\right)_{+}>0$ (otherwise we may omit the summand $\left.\left(W_{s_{i}}-W_{t_{i}}-c\right)_{+}\right)$. From the definition of $T_{c}$ we have that for any $1 \leq i \leq n_{0}-1, W_{s_{i}}-W_{t_{i+1}}<c$, so

$$
\begin{aligned}
& \left(W_{s_{i}}-W_{t_{i}}-c\right)_{+}+\left(W_{s_{i+1}}-W_{t_{i+1}}-c\right)_{+} \\
& =W_{s_{i}}-W_{t_{i}}-c+W_{s_{i+1}}-W_{t_{i+1}}-c \\
& =W_{s_{i+1}}-W_{t_{i}}-c+\left(W_{s_{i}}-W_{t_{i+1}}-c\right)<W_{s_{i+1}}-W_{t_{i}}-c .
\end{aligned}
$$

Iterating the above inequality, we obtain

$$
\sum_{i=1}^{n_{0}}\left(W_{s_{i}}-W_{t_{i}}-c\right)_{+} \leq W_{s_{n_{0}}}-W_{t_{1}}-c \leq \sup _{0 \leq t<s \leq T_{c} \wedge T}\left(W_{s}-W_{t}-c\right)_{+} .
$$

This, together with the obvious inequality

$$
\sum_{i=n_{0}+1}^{n}\left(W_{s_{i}}-W_{t_{i}}-c\right)_{+} \leq U T V_{\mu}^{c}\left[T_{c}, T\right]
$$

proves (5). Taking the supremum over all partitions $0 \leq t_{1}<s_{1}<t_{2}<s_{2}<\cdots<t_{n}<$ $s_{n} \leq T$ we finally get

$$
U T V_{\mu}^{c}[0, T] \leq \sup _{0 \leq t<s \leq T_{c} \wedge T}\left(W_{s}-W_{t}-c\right)_{+}+U T V_{\mu}^{c}\left[T_{c}, T\right] .
$$

Since the opposite inequality is obvious, we finally get (4).

Let us now define some auxiliary variables. Let $T_{c}^{(0)} \equiv 0$ and let $T_{c}^{(i)}, i=1,2, \ldots$ be defined recursively as

$$
T_{c}^{(i)}=\inf \left\{t>T_{c}^{(i-1)}: \sup _{T_{c}^{(i-1)} \leq s \leq t} W_{s} \geq W_{t}+c\right\} .
$$


(notice that $T_{c}^{(1)}=T_{c}$ ). We define a new variable

$$
U T V_{\mu}^{c}(T):=\sum_{i=1}^{\infty} e^{-T_{c}^{(i-1)} / T} \sup _{T_{c}^{(i-1)} \leq t<s \leq T_{c}^{(i)} \wedge\left(T_{c}^{(i-1)}+T\right)}\left(W_{s}-W_{t}-c\right)_{+} \cdot
$$

We have the following

Lemma 3.2. The variables $U T V_{\mu}^{c}[0, T]$ and $U T V_{\mu}^{c}(T)$ are related by the following relations

$$
\begin{gathered}
U T V_{\mu}^{c}[0, T] \leq e U T V_{\mu}^{c}(T), \\
U T V_{\mu}^{c}[0, T] \succeq \frac{1-e^{-1}}{2} U T V_{\mu}^{c}(T),
\end{gathered}
$$

where the first relation holds almost surely and the second holds in the sense of stochastic domination i.e. for every $y \geq 0, P\left(U T V_{\mu}^{c}[0, T] \geq y\right) \geq P\left(\frac{1-e^{-1}}{2} U T V_{\mu}^{c}(T) \geq y\right)$.

Proof. By the previous lemma, we have

$$
\begin{aligned}
& U T V_{\mu}^{c}[0, T]=\sup _{0 \leq t<s \leq T_{c}^{(1)} \wedge T}\left(W_{s}-W_{t}-c\right)_{+}+U T V_{\mu}^{c}\left[T_{c}^{(1)}, T\right] \\
& =\sup _{0 \leq t<s \leq T_{c}^{(1)} \wedge T}\left(W_{s}-W_{t}-c\right)_{+}+\sup _{T_{c}^{(1)} \leq t<s \leq T_{c}^{(2)} \wedge T}\left(W_{s}-W_{t}-c\right)_{+} \\
& +U T V_{\mu}^{c}\left[T_{c}^{(2)}, T\right] \\
& =\ldots=\sum_{i \geq 1: T_{c}^{(i-1)} \leq T} \sup _{T_{c}^{(i-1)} \leq t<s \leq T_{c}^{(i)} \wedge T}\left(W_{s}-W_{t}-c\right)_{+} .
\end{aligned}
$$

From 11 we almost immediately get $(9)$

$$
\begin{aligned}
U T V_{\mu}^{c}[0, T] & =\sum_{i \geq 1: T_{c}^{(i-1)} \leq T} \sup _{\substack{T_{c}^{(i-1)} \leq t<s \leq T_{c}^{(i)} \wedge T\\
}}\left(W_{s}-W_{t}-c\right)_{+} \\
& \leq \sum_{i=1}^{\infty} e^{1-T_{c}^{(i-1)} / T} \sup _{T_{c}^{(i-1)} \leq t<s \leq T_{c}^{(i)} \wedge\left(T_{c}^{(i-1)}+T\right)}\left(W_{s}-W_{t}-c\right)_{+} \\
& =e U T V_{\mu}^{c}(T) .
\end{aligned}
$$

In order to prove the second relation let $i_{0} \geq 1$ be the greatest index such that $T_{c}^{\left(i_{0}-1\right)}<T$ and let us consider the term

$$
A=\sup _{T_{c}^{\left(i_{0}-1\right)} \leq t<s \leq T_{c}^{\left(i_{0}\right)} \wedge\left(T_{c}^{\left(i_{0}-1\right)}+T\right)}\left(W_{s}-W_{t}-c\right)_{+} \cdot
$$

If $i_{0}=1$ then $A=\sup _{0 \leq t<s \leq T_{c}^{(1)} \wedge T}\left(W_{s}-W_{t}-c, 0\right)_{+}$, otherwise $A$ is independent of $B=\sup _{0 \leq t<s \leq T_{c}^{(1)} \wedge T}\left(W_{s}-W_{t}-c, 0\right)_{+}$but has the same distribution as $B$. 
By (11) we have

$$
\begin{aligned}
U T V_{\mu}^{c}[0, T]= & \sum_{i \geq 1: T_{c}^{(i-1)} \leq T} \sup _{T_{c}^{(i-1)} \leq t<s \leq T_{c}^{(i)} \wedge T}\left(W_{s}-W_{t}-c\right)_{+} \\
= & \sum_{i=1}^{i_{0}-1} \sup _{T_{c}^{(i-1)} \leq t<s \leq T_{c}^{(i)}}\left(W_{s}-W_{t}-c\right)_{+} \\
& +\sup _{T_{c}^{\left(i_{0}-1\right)} \leq t<s \leq T}\left(W_{s}-W_{t}-c\right)_{+} .
\end{aligned}
$$

In both cases $\left(i_{0}=1\right.$ and $\left.i_{0}>1\right) 2 U T V_{\mu}^{c}[0, T]$ stochastically dominates the sum

$$
S_{1}=\sum_{i=1}^{i_{0}} e^{-T_{c}^{(i-1)} / T} \sup _{T_{c}^{(i-1)} \leq t<s \leq T_{c}^{(i)} \wedge\left(T_{c}^{(i-1)}+T\right)}\left(W_{s}-W_{t}-c\right)_{+} \cdot
$$

$\left(\sum_{i=1}^{i_{0}-1} \sup _{T_{c}^{(i-1)} \leq t<s \leq T_{c}^{(i)}}\left(W_{s}-W_{t}-c\right)_{+}\right.$dominates the first $i_{0}-1$ terms in the above sum and $B$, which appears in the sum $\left(12\right.$ dominates $A$.) Similarly, define $i_{k}$ recursively as the greatest integer such that $T_{c}^{\left(i_{k}-1\right)}<T_{c}^{\left(i_{k-1}\right)}+T$ and

$$
S_{k}=\sum_{i=i_{k-1}+1}^{i_{k}} \exp \left(-\frac{T_{c}^{(i-1)}-T_{c}^{\left(i_{k-1}\right)}}{T}\right)_{T_{c}^{(i-1)} \leq t<s \leq T_{c}^{(i)} \wedge\left(T_{c}^{(i-1)}+T\right)}\left(W_{s}-W_{t}-c\right)_{+} \cdot
$$

$S_{k}$ is independent of $S_{1}, \ldots, S_{k-1}$, moreover it has the same distribution as $S_{1}$ and

$$
U T V_{\mu}^{c}(T)=\sum_{k=1}^{\infty} e^{-T_{c}^{\left(i_{k-1}\right)} / T} S_{k}
$$

By the definition of $i_{k}, T_{c}^{\left(i_{k}\right)} \geq T_{c}^{\left(i_{k-1}\right)}+T$, thus we have $T_{c}^{\left(i_{k}\right)} \geq(k-1) T$. Now, since $2 U T V_{\mu}^{c}[0, T] \succeq S_{k}, k=1,2, \ldots$, we have that

$$
\begin{aligned}
\frac{2}{1-e^{-1}} U T V_{\mu}^{c}[0, T] & =\sum_{k=1}^{\infty} e^{-(k-1)} 2 U T V_{\mu}^{c}[0, T] \\
& \succeq \sum_{k=1}^{\infty} e^{-T_{c}^{\left(i_{k}-1\right)} / T} 2 U T V_{\mu}^{c}[0, T] \\
& \succeq \sum_{k=1}^{\infty} e^{-T_{c}^{\left(i_{k}-1\right)} / T} S_{k}=U T V_{\mu}^{c}(T) .
\end{aligned}
$$

which proves 10 .

Next, let us state a refinement of Lemma 3 from [6]:

Lemma 3.3. For any $\mu$ and $c>0$

$$
P\left(T_{c}<\frac{1}{3} \mathbf{E} T_{c}\right) \leq \frac{7}{9}
$$

Proof. The proof follows exactly as in [6], since one can show that for any real $\mu$

$$
\frac{\left(\mathbf{E} T_{c}\right)^{2}}{\mathbf{E} T_{c}^{2}}=\frac{1}{2} \frac{\left(e^{2 \mu c}-1-2 \mu c\right)^{2}}{e^{4 \mu c}-6 e^{2 \mu c} \mu c+e^{2 \mu c}+2 \mu^{2} c^{2}-2} \geq \frac{1}{2}
$$


and, by the Paley-Zygmund inequality we obtain

$$
P\left(T_{c} \geq \frac{1}{3} \mathbf{E} T_{c}\right) \geq\left(1-\frac{1}{3}\right)^{2} \frac{\left(\mathbf{E} T_{c}\right)^{2}}{\mathbf{E} T_{c}^{2}} \geq \frac{4}{9} \frac{1}{2}=\frac{2}{9}
$$

and

$$
P\left(T_{c}<\frac{1}{3} \mathbf{E} T_{c}\right)=1-P\left(T_{c} \geq \frac{1}{3} \mathbf{E} T_{c}\right) \leq \frac{7}{9}
$$

3.2. Estimates for long and short time intervals. Now we are ready to prove estimates of the expected value of $U T V_{\mu}^{c}[0, T]$ for long and short time intervals $\left(T \geq \frac{1}{3} \mathbf{E} T_{c}\right.$ and $T<\frac{1}{3} \mathbf{E} T_{c}$ respectively). We have

Theorem 3.4. For any $T \geq \frac{1}{3} \mathbf{E} T_{c}$ we have

$$
\begin{aligned}
0.3 \frac{T}{\mathbf{E} T_{c}} \mathbf{E} \sup _{0 \leq t<s \leq T_{c} \wedge T}\left(W_{s}-W_{t}-c\right)_{+} & \leq \mathbf{E} U T V_{\mu}^{c}[0, T] \\
& \leq 27 \frac{T}{\mathbf{E} T_{c}} \mathbf{E} \sup _{0 \leq t<s \leq T_{c} \wedge T}\left(W_{s}-W_{t}-c\right)_{+} .
\end{aligned}
$$

Proof. By Lemma 3.1 and independence of $W_{t}-W_{T_{c}}, t \geq T_{c}$, and $T_{c}$ (strong Markov property of Brownian motion) we calculate

$$
\begin{aligned}
\mathbf{E} U T V_{\mu}^{c}[0, T]= & \mathbf{E} \sup _{0 \leq t \leq s \leq T_{c} \wedge T}\left(W_{s}-W_{t}-c\right)_{+}+\mathbf{E} U T V_{\mu}^{c}\left[T_{c} \wedge T, T\right] \\
\leq & \mathbf{E} \sup _{0 \leq t<s \leq T_{c} \wedge T}\left(W_{s}-W_{t}-c\right)_{+}+\mathbf{E}\left[U T V_{\mu}^{c}\left[T_{c}, T\right] ; T_{c}<\frac{1}{3} \mathbf{E} T_{c}\right] \\
& +\mathbf{E}\left[U T V_{\mu}^{c}\left[T_{c}, T\right] ; \frac{1}{3} \mathbf{E} T_{c} \leq T_{c} \leq T\right] \\
\leq & \mathbf{E} \sup _{0 \leq t<s \leq T_{c} \wedge T}\left(W_{s}-W_{t}-c\right)_{+}+\mathbf{E}\left[U T V_{\mu}^{c}\left[T_{c}, T+T_{c}\right] ; T_{c}<\frac{1}{3} \mathbf{E} T_{c}\right] \\
& +\mathbf{E}\left[U T V_{\mu}^{c}\left[T_{c}, T+T_{c}-\frac{1}{3} \mathbf{E} T_{c}\right] ; \frac{1}{3} \mathbf{E} T_{c} \leq T_{c} \leq T\right] \\
\leq & \mathbf{E} \sup _{0 \leq t<s \leq T_{c} \wedge T}\left(W_{s}-W_{t}-c\right)_{+}+\mathbf{E} U T V_{\mu}^{c}[0, T] P\left(T_{c}<\frac{1}{3} \mathbf{E} T_{c}\right) \\
& +\mathbf{E} U T V_{\mu}^{c}\left[0, T-\frac{1}{3} \mathbf{E} T_{c}\right] P\left(T_{c} \geq \frac{1}{3} \mathbf{E} T_{c}\right) .
\end{aligned}
$$

Now, by the above inequality and Lemma 3.3

$$
\begin{aligned}
\mathbf{E} U T V_{\mu}^{c}[0, T] & \leq \frac{\mathbf{E} \sup _{0 \leq t<s \leq T_{c} \wedge T}\left(W_{s}-W_{t}-c\right)_{+}}{P\left(T_{c} \geq \frac{1}{3} \mathbf{E} T_{c}\right)}+\mathbf{E} U T V_{\mu}^{c}\left[0, T-\frac{1}{3} \mathbf{E} T_{c}\right] \\
& \leq \frac{9}{2} \mathbf{E} \sup _{0 \leq t<s \leq T_{c} \wedge T}\left(W_{s}-W_{t}-c\right)_{+}+\mathbf{E} U T V_{\mu}^{c}\left[0, T-\frac{1}{3} \mathbf{E} T_{c}\right] .
\end{aligned}
$$

Similarly

$$
\mathbf{E} U T V_{\mu}^{c}\left[0, T-\frac{1}{3} \mathbf{E} T_{c}\right] \leq \frac{9}{2} \mathbf{E} \sup _{0 \leq t<s \leq T_{c} \wedge T}\left(W_{s}-W_{t}-c\right)_{+}+\mathbf{E} U T V_{\mu}^{c}\left[0, T-\frac{2}{3} \mathbf{E} T_{c}\right] .
$$


Iterating and putting together the above inequalities we obtain the estimate from above

$$
\begin{aligned}
\mathbf{E} U T V_{\mu}^{c}[0, T] & \leq\left\lceil\frac{T}{\frac{1}{3} \mathbf{E} T_{c}}\right] \frac{9}{2} \mathbf{E} \sup _{0 \leq t \leq s \leq T_{c} \wedge T}\left(W_{s}-W_{t}-c\right)_{+} \\
& \leq\left(\frac{3 T}{\mathbf{E} T_{c}}+1\right) \frac{9}{2} \mathbf{E} \sup _{0 \leq t<s \leq T_{c} \wedge T}\left(W_{s}-W_{t}-c\right)_{+} \\
& \leq \frac{6 T}{\mathbf{E} T_{c}} \frac{9}{2} \mathbf{E} \sup _{0 \leq t \leq s \leq T_{c} \wedge T}\left(W_{s}-W_{t}-c\right)_{+} \\
& \leq 27 \frac{T}{\mathbf{E} T_{c}} \mathbf{E} \sup _{0 \leq t \leq s \leq T_{c} \wedge T}\left(W_{s}-W_{t}-c\right)_{+} .
\end{aligned}
$$

The estimate from below is obtained from Lemma 3.2 (see also the comment after the calculation):

$$
\begin{aligned}
& \mathbf{E} U T V_{\mu}^{c}[0, T] \geq \frac{1-e^{-1}}{2} \mathbf{E} U T V_{\mu}^{c}(T) \geq 0.3 \mathbf{E} U T V_{\mu}^{c}(T) \\
& =0.3 \sum_{i=1}^{\infty} \mathbf{E} e^{-T_{c}^{(i-1)} / T} \sup _{T_{c}^{(i-1)} \leq t<s \leq T_{c}^{(i)} \wedge\left(T_{c}^{(i-1)}+T\right)}\left(W_{s}-W_{t}-c\right)_{+} \\
& =0.3 \sum_{i=1}^{\infty} \mathbf{E} e^{-T_{c}^{(i-1)} / T} \mathbf{E} \sup _{T_{c}^{(i-1)} \leq t<s \leq T_{c}^{(i)} \wedge\left(T_{c}^{(i-1)}+T\right)}\left(W_{s}-W_{t}-c\right)_{+} \\
& =0.3\left(\sum_{i=1}^{\infty}\left(\mathbf{E} e^{-T_{c}^{(1)} / T}\right)^{i-1}\right) \mathbf{E} \sup _{0 \leq t \leq s \leq T_{c} \wedge T}\left(W_{s}-W_{t}-c\right)_{+} \\
& =0.3 \frac{1}{1-\mathbf{E} e^{-T_{c}^{(1)} / T}} \mathbf{E} \sup _{0 \leq t<s \leq T_{c} \wedge T}\left(W_{s}-W_{t}-c\right)_{+} \\
& \geq 0.3 \frac{1}{1-\mathbf{E}\left(1-T_{c}^{(1)} / T\right)} \mathbf{E} \sup _{0 \leq t<s \leq T_{c} \wedge T}\left(W_{s}-W_{t}-c\right)_{+} \\
& =0.3 \frac{T}{\mathbf{E} T_{c}} \mathbf{E} \sup _{0 \leq t \leq s \leq T_{c} \wedge T}\left(W_{s}-W_{t}-c\right)_{+} \text {. }
\end{aligned}
$$

In the above calculations we used consecutively: the independence of $T_{c}^{(i-1)}$ and $W_{s}-$ $W_{T_{c}^{(i-1)}}, s \geq T_{c}^{(i-1)}$, the equality of distributions of every term

$$
\sup _{T_{c}^{(i-1)} \leq t<s \leq T_{c}^{(i)} \wedge\left(T_{c}^{(i-1)}+T\right)}\left(W_{s}-W_{t}-c\right)_{+}
$$

for $i=1,2, \ldots$, the definition of $T_{c}^{(i-1)}$, which implies the equality

$$
\mathbf{E} e^{-T_{c}^{(i-1)} / T}=\left(\mathbf{E} e^{-T_{c}^{(1)} / T}\right)^{i-1},
$$

and finally we used the inequality $e^{x} \geq 1+x$.

The estimates in Theorem 3.4 involve the expected value of the variable

$$
\sup _{0 \leq t<s \leq T_{c} \wedge T}\left(W_{s}-W_{t}-c\right)_{+}
$$

the distribution of which, as far as the author knows, is not known, but it may be simulated numerically. We also have 
Corollary 3.5. For any $T \geq \frac{1}{3} \mathbf{E} T_{c}$ we have

$$
\begin{aligned}
3 \frac{T}{\mathbf{E} T_{c}} \mathbf{E} \sup _{0 \leq t \leq s \leq \frac{1}{3} \mathbf{E} T_{c}}\left(W_{s}-W_{t}-c\right)_{+} & \leq \mathbf{E} U T V_{\mu}^{c}[0, T] \\
& \leq 27 \frac{T}{\mathbf{E} T_{c}} \mathbf{E} \sup _{0 \leq t \leq s \leq T_{c}}\left(W_{s}-W_{t}-c\right)_{+} .
\end{aligned}
$$

Proof. The estimate from above is a straightforward consequence of Theorem 3.4 and the estimate from below is obtained immediately by superadditivity:

$$
\begin{aligned}
\mathbf{E} U T V_{\mu}^{c}[0, T] & \geq \sum_{i=1}^{\left\lfloor 3 T / \mathbf{E} T_{c}\right\rfloor} \mathbf{E} U T V_{\mu}^{c}\left[\frac{i-1}{3} \mathbf{E} T_{c}, \frac{i}{3} \mathbf{E} T_{c}\right] \\
& \geq\left\lfloor 3 T / \mathbf{E} T_{c}\right\rfloor \mathbf{E} U T V_{\mu}^{c}\left[0, \frac{1}{3} \mathbf{E} T_{c}\right] \\
& \geq 3 \frac{T}{\mathbf{E} T_{c}} \mathbf{E} \sup _{0 \leq t \leq s \leq \frac{1}{3} \mathbf{E} T_{c}}\left(W_{s}-W_{t}-c\right)_{+} .
\end{aligned}
$$

REMARK. Using the results of Hadjiliadis and Vecer [1] we are able to calculate exactly the estimate from above appearing in [13). Using the notation from [1, for $z>0$ we have

$$
\begin{aligned}
P\left(\sup _{0 \leq t \leq s \leq T_{c}}\left(W_{s}-W_{t}-c\right)_{+} \geq z\right) & =P\left(\sup _{0 \leq t \leq s \leq T_{c}}\left(W_{s}-W_{t}\right) \geq z+c\right) \\
& =P\left(T(c, z+c)=T_{2}(z+c)\right)
\end{aligned}
$$

and by Theorem 2.1 from [1], for $y>c$ we have

$$
P\left(\sup _{0 \leq t \leq s \leq T_{c}}\left(W_{s}-W_{t}\right) \geq y\right)=\frac{e^{2 \mu c}-2 \mu c-1}{e^{2 \mu c}+e^{-2 \mu c}-2} \exp \left(-\frac{2 \mu}{e^{2 \mu c}-1}(y-c)\right) .
$$

Hence

$$
\begin{aligned}
\mathbf{E} \sup _{0 \leq t \leq s \leq T_{c}}\left(W_{s}-W_{t}-c\right)_{+} & =\int_{c}^{\infty} P\left(\sup _{0 \leq t \leq s \leq T_{c}}\left(W_{s}-W_{t}\right) \geq y\right) d y \\
& =\frac{e^{2 \mu c}-2 \mu c-1}{e^{2 \mu c}+e^{-2 \mu c}-2} \int_{c}^{\infty} \exp \left(-\frac{2 \mu}{e^{2 \mu c}-1}(y-c)\right) d y \\
& =\frac{e^{2 \mu c}-2 \mu c-1}{e^{2 \mu c}+e^{-2 \mu c}-2} \frac{e^{2 \mu c}-1}{2 \mu} .
\end{aligned}
$$

Estimates of $\mathbf{E} U T V_{\mu}^{c}[0, T]$ for short time intervals $\left(T<\frac{1}{2} \mathbf{E} T_{c}\right)$ are the subject of the next theorem.

THEOREM 3.6. For any $T<\frac{1}{3} \mathbf{E} T_{c}$ we have

$$
\begin{aligned}
\mathbf{E} \sup _{0 \leq t \leq s \leq T}\left(W_{s}-W_{t}-c\right)_{+} & \leq \mathbf{E} U T V_{\mu}^{c}[0, T] \\
& \leq 5 \mathbf{E} \sup _{0 \leq t \leq s \leq T}\left(W_{s}-W_{t}-c\right)_{+} .
\end{aligned}
$$

Proof. Applying Lemma 3.1 and the independence of $W_{t}-W_{T_{c}}, t \geq T_{c}$, and $T_{c}$ we again 
calculate

$$
\begin{aligned}
\mathbf{E} U T V_{\mu}^{c}[0, T] & \leq \mathbf{E} \sup _{0 \leq t \leq s \leq T_{c} \wedge T}\left(W_{s}-W_{t}-c\right)_{+}+\mathbf{E} U T V_{\mu}^{c}\left[T_{c} \wedge T, T\right] \\
& \leq \mathbf{E} \sup _{0 \leq t \leq s \leq T}\left(W_{s}-W_{t}-c\right)_{+}+\mathbf{E}\left[U T V_{\mu}^{c}\left[T_{c}, T\right] ; T_{c}<T\right] \\
& \leq \mathbf{E} \sup _{0 \leq t \leq s \leq T}\left(W_{s}-W_{t}-c\right)_{+}+\mathbf{E} U T V_{\mu}^{c}[0, T] P\left(T_{c}<\frac{1}{3} \mathbf{E} T_{c}\right) \\
& \leq \mathbf{E} \sup _{0 \leq t \leq s \leq T}\left(W_{s}-W_{t}-c\right)_{+}+\mathbf{E} U T V_{\mu}^{c}[0, T] \frac{7}{9} .
\end{aligned}
$$

Thus we get

$$
\mathbf{E} U T V_{\mu}^{c}[0, T] \leq \frac{9}{2} \mathbf{E} \sup _{0 \leq t \leq s \leq T}\left(W_{s}-W_{t}-c\right)_{+} \cdot
$$

The estimate from above is self-evident

$$
\mathbf{E} U T V_{\mu}^{c}[0, T] \geq \mathbf{E} \sup _{0 \leq t \leq s \leq T}\left(W_{s}-W_{t}-c\right)_{+} .
$$

REMARK. In order to calculate the quantity $\mathbf{E} \sup _{0 \leq t \leq s \leq T}\left(W_{s}-W_{t}-c\right)_{+}$for $T \leq \frac{1}{3} \mathbf{E} T_{c}$, which appears in Corollary 3.5 and in Theorem 3.6, one may use the results of [5]. Let

$$
G_{\bar{D}}(y)=2 e^{\mu y}\left\{L+\sum_{n=1}^{\infty} \frac{\theta_{n} \sin \theta_{n}}{\theta_{n}^{2}+\mu^{2} y^{2}+\mu y}\left(1-\exp \left(-\frac{\theta_{n}^{2} T}{2 y^{2}}-\frac{\mu^{2} T}{2}\right)\right)\right\},
$$

where $\theta_{n}$ are positive solutions of the eigenvalue condition $\tan \theta_{n}=-\frac{\theta_{n}}{\mu y}$,

$$
L=\left\{\begin{array}{c}
0,0<y<-\frac{1}{\mu} \\
\frac{3}{2}\left(1-e^{-\mu^{2} T / 2}\right), y=-\frac{1}{\mu} \\
\frac{2 \eta \sinh \eta}{\eta^{2}-\mu^{2} y^{2}-\mu y}\left(1-\exp \left(\frac{\eta^{2} T}{2 y^{2}}-\frac{\mu^{2} T}{2}\right)\right), y>-\frac{1}{\mu}
\end{array}\right.
$$

and $\eta$ is the unique positive solution of $\tanh \eta=-\frac{\eta}{\mu y}$. In the notation used in [5] for $z>0$ we have

$$
\begin{aligned}
P\left(\sup _{0 \leq t \leq s \leq T}\left(W_{s}-W_{t}-c\right)_{+} \geq z\right) & =P\left(\sup _{0 \leq t \leq s \leq T}\left(W_{s}-W_{t}\right) \geq z+c\right) \\
& =P(\bar{D}(T ;-\mu, 1) \geq z+c)=G_{\bar{D}}(z+c)
\end{aligned}
$$

and thus

$$
\mathbf{E} \sup _{0 \leq t \leq s \leq T}\left(W_{s}-W_{t}-c\right)_{+}=\int_{0}^{\infty} G_{\bar{D}}(z+c) d z=\int_{c}^{\infty} G_{\bar{D}}(z) d z .
$$

However, the above formula is numerically very unstable and it does not seem to be a straightforward task to apply it to obtain good numerical or analytical estimates of the expected value of the variable $\sup _{0 \leq t \leq s \leq T}\left(W_{s}-W_{t}-c\right)_{+}$.

4. Example of application. As mentioned earlier, upward truncated variation appears naturally in the expression for the least upper bound for the rate of return from any trading of a financial asset, the dynamics of which follows geometric Brownian motion, in the presence of flat commission. A similar result was proved in [6] for truncated variation, however, truncated variation is not the least upper bound. 
Indeed, similarly as in [6], let us assume that the dynamics of the prices $P_{t}$ of some financial asset (e.g. stock) is the following $P_{t}=\exp \left(\mu t+\sigma B_{t}\right)$. We are interested in the maximal possible profit coming from trading this single instrument during the time interval $[0, T]$. We buy the instrument at times $0 \leq t_{1}<\cdots<t_{n}<T$ and sell it at $s_{1}<\cdots<s_{n} \leq T$, where $t_{1}<s_{1}<t_{2}<s_{2}<\cdots<t_{n}<s_{n}$, in order to obtain the maximal possible profit. Furthermore we assume that for every transaction we have to pay a flat commission and $\gamma$ is the ratio of the transaction value paid for the commission.

The maximal possible rate of return from our strategy is (cf. [6])

$$
\sup _{n} \sup _{0 \leq t_{1}<s_{1}<\cdots<t_{n}<s_{n} \leq T} \frac{P_{s_{1}}}{P_{t_{1}}} \frac{1-\gamma}{1+\gamma} \cdots \frac{P_{s_{n}}}{P_{t_{n}}} \frac{1-\gamma}{1+\gamma}-1 .
$$

Let $M_{n}$ be the set of all partitions

$$
\pi=\left\{0 \leq t_{1}<s_{1}<\cdots<t_{n}<s_{n} \leq T\right\} .
$$

To see that $\exp \left(\sigma U T V_{\mu / \sigma}^{c / \sigma}[0, T]\right)-1$ with $c=\ln \frac{1+\gamma}{1-\gamma}$ is the least upper bound for maximal possible rate of return let us calculate

$$
\begin{aligned}
& \sup _{n} \sup _{M_{n}} \prod_{i=1}^{n}\left\{\frac{P_{s_{i}}}{P_{t_{i}}} \frac{1-\gamma}{1+\gamma}\right\}=\sup _{n} \sup _{M_{n}} \prod_{i=1}^{n}\left\{\frac{\exp \left(\mu s_{i}+\sigma B_{s_{i}}\right)}{\exp \left(\mu t_{i}+\sigma B_{t_{i}}\right)} e^{-c}\right\} \\
& =\sup _{n} \sup _{M_{n}} \exp \left(\sigma \sum_{i=1}^{n}\left\{\left(\frac{\mu}{\sigma} s_{i}+B_{s_{i}}\right)-\left(\frac{\mu}{\sigma} t_{i}+B_{t_{i}}\right)-\frac{c}{\sigma}\right\}\right) \\
& =\exp \left(\underset{n \sup _{n} M_{n}}{\sigma} \sum_{i=1}^{n}\left\{\left(\frac{\mu}{\sigma} s_{i}+B_{s_{i}}\right)-\left(\frac{\mu}{\sigma} t_{i}+B_{t_{i}}\right)-\frac{c}{\sigma}\right\}\right) \\
& =\exp \left(\sigma U T V_{\mu / \sigma}^{c / \sigma}[0, T]\right) .
\end{aligned}
$$

This gives the claimed bound.

\section{References}

[1] O. Hadjiliadis and J. Vecer, Drawdowns preceding rallies in the Brownian motion model, Quantitative Finance 6 (2006), no. 5, 403-409.

[2] Z. J. Jurek, A limit theorem for truncated random variables, Bull. Pol. Acad. Sci. Math. 23 (1975), no. 8, 911-916.

[3] Z. J. Jurek, Relations between the s-selfdecomposable and selfdecomposable measures, Ann. Probab. 13 (1985), no. 2, 592-608.

[4] A. M. Iksanov, Z. J. Jurek and B. M. Schreiber, A new factorization property of selfdecomposable probability measures, Ann. Probab. 32 (2004), no. 2, 1356-1369.

[5] M. Magdon-Ismail, A. F. Atiya, A. Pratap and Y. S. Abu-Mostafa, On the maximum drawdown of a Brownian motion, J. Appl. Probability 41 (2004), 147-161.

[6] R. Łochowski, On truncated variation of Brownian motion with drift, Bull. Pol. Acad. Sci. Math. 56 (2008), no. 4, 267-281. 